\title{
Implantable Therapeutic Reservoir Systems for Diverse Clinical Applications in Large Animal Models
}

\author{
Garry P. Duffy, Scott T. Robinson, Raymond O'Connor, Robert Wylie, \\ Ciaran Mauerhofer, Gabriella Bellavia, Stefania Straino, Francesca Cianfarani, \\ Keegan Mendez, Rachel Beatty, Ruth Levey, Janice O'Sullivan, Liam McDonough, \\ Helena Kelly, Ellen T. Roche,* and Eimear B. Dolan*
}

Regenerative medicine approaches, specifically stem cell technologies, have demonstrated significant potential to treat a diverse array of pathologies. However, such approaches have resulted in a modest clinical benefit, which may be attributed to poor cell retention/survival at the disease site. A delivery system that facilitates regional and repeated delivery to target tissues can provide enhanced clinical efficacy of cell therapies when localized delivery of high doses of cells is required. In this study, a new regenerative reservoir platform (Regenervoir) is described for use in large animal models, with relevance to cardiac, abdominal, and soft tissue pathologies. Regenervoir incorporates multiple novel design features essential for clinical translation, with a focus on scalability, mechanism of delivery, fixation to target tissue, and filling/refilling with a therapeutic cargo, and is demonstrated in an array of clinical applications that are easily translated to human studies. Regenervoir consists of a porous reservoir fabricated from a single material, a flexible thermoplastic polymer, capable of delivering cargo via fill lines to target tissues. A radiopaque shear thinning hydrogel can be delivered to the therapy reservoir and multiple fixation methods (laparoscopic tacks and cyanoacrylate bioadhesive) can be used to secure Regenervoir to target tissues through a minimally invasive approach.

\section{Introduction}

Cell therapy encompasses a broad clinical territory in which transplanted cells are used to terminate or reverse an assortment of disease processes, such as cardiovascular disease, ${ }^{[1,2]}$ diabetes, ${ }^{[3]}$ autoimmune disorders, ${ }^{[4]}$ neurodegenerative disease, ${ }^{[5,6]}$ and cancer. ${ }^{[7]}$ The last decade has seen an exponential advancement of cell therapy technologies, with promising preclinical results finally yielding substantial clinical benefit using Chimeric Antigen Receptor T-cell (CAR T) for treatment of hematologic malignancies. ${ }^{\left[{ }^{8-10]}\right.}$ However, despite these isolated clinical successes, other cell therapy approaches have provided only a modest clinical benefit, which may be attributed to poor cell retention and survival at the disease site. ${ }^{[11-13]}$

A delivery system that facilitates regional and repeated delivery to target
Prof. G. P. Duffy, Dr. S. T. Robinson, R. O'Connor, R. Wylie,

C. Mauerhofer, R. Beatty, R. Levey, Dr. J. O'Sullivan, Dr. E. B. Dolan

Anatomy \& Regenerative Medicine Institute (REMEDI)

School of Medicine, College of Medicine Nursing and Health Sciences

National University of Ireland

Galway H91 W5P7, Ireland

E-mail: Eimear.dolan@nuigalway.ie

Prof. G. P. Duffy, Dr. S. T. Robinson, R. Beatty

Advanced Materials and BioEngineering Research Centre (AMBER)

Trinity College Dublin

Dublin D02 PN40, Ireland

Prof. G. P. Duffy

CÚRAM, Centre for Research in Medical Devices

National University of Ireland Galway

Galway H91 TK33, Ireland

Dr. S. T. Robinson

Department of Surgery

University of Michigan

Ann Arbor, MI 48109, USA

Dr. G. Bellavia, S. Straino, Dr. F. Cianfarani

Explora Biotech Srl

G. Peroni 386, Rome 00131, Italy

The ORCID identification number(s) for the author(s) of this article can be found under https://doi.org/10.1002/adhm.202000305.

DOI: 10.1002/adhm.202000305

\author{
K. Mendez, Prof. E. T. Roche \\ Institute for Medical Engineering and Science \\ Massachusetts Institute of Technology \\ Cambridge, MA 02139, USA \\ E-mail: etr@mit.edu \\ K. Mendez, Prof. E. T. Roche \\ Harvard-MIT Program in Health Sciences and Technology \\ Cambridge, MA 02139, USA \\ Dr. L. McDonough, Prof. H. Kelly \\ School of Pharmacy and Molecular Sciences \\ Royal College of Surgeons in Ireland \\ 111 St. Stephen's Green, Dublin 2, D02 VN51, Ireland \\ Dr. L. McDonough, Prof. H. Kelly \\ Tissue Engineering Research Group \\ Department of Anatomy \\ Royal College of Surgeons in Ireland \\ 123 St. Stephen's Green, Dublin 2, D02 YN77, Ireland \\ Prof. E. T. Roche \\ Department of Mechanical Engineering \\ Massachusetts Institute of Technology \\ Cambridge, MA 02139, USA \\ Dr. E. B. Dolan \\ Department of Biomedical Engineering \\ School of Engineering, College of Science and Engineering \\ National University of Ireland \\ Galway H91 TK33, Ireland
}


tissues can provide enhanced clinical efficacy of cell therapies when localized delivery of a high dose of cells is required. For example, direct delivery of cells to the intraperitoneal space can improve clinical benefit compared to systemic intravenous administration. Stem cells delivered to the intraperitoneal space show a substantial preclinical benefit in treatment of inflammatory bowel disease ${ }^{[14,15]}$ and rheumatoid arthritis ${ }^{[16,17]}$ compared to intravenous therapy. Intraperitoneal infusion with CAR T-cells can also reduce tumor burden in animal models of peritoneal carcinomatosis, ${ }^{[18]}$ with repeat dosing leading to an improved response. ${ }^{[19,20]}$ Intramuscular delivery of cells to skeletal muscle has also been used for treatment of critical limb ischemia ${ }^{[21-24]}$ and skeletal muscle regeneration, ${ }^{[25-27]}$ obviating the need for localized delivery of cells to intramuscular targets. Cardiac tissue may also benefit from direct cell delivery compared to systemic administration; for example, implantation of stem cells in the heart leads to improved retention ${ }^{[28]}$ and cardiac function after myocardial infarction via paracrine mediators, ${ }^{[11-13]}$ and several studies in rodents suggest repeated dosing provides functional improvement compared to a single infusion of cells. ${ }^{[29-33]}$

In order to address the need for local, repeated delivery of cells after myocardial infarction, we previously developed a refillable, flexible polymer reservoir that enabled delivery of multiple doses of mesenchymal stem cells (MSCs) to ischemic cardiac tissue in a small animal model of acute myocardial infarction. Repeated dosing of MSCs resulted in improved ejection fraction and fractional shortening, and established the therapeutic value of an implantable reservoir that permits repeated dosing of cellular cargo. ${ }^{[33]}$ No such device exists for large animal models.

In this report, we expand upon this earlier work with a feasibility study where a new regenerative reservoir platform (Regenervoir) is designed and manufactured for use in three large animal models that are easily translated to human studies, a significant leap forward. The Regenervoir system was designed for use in a diverse set of clinical applications, and incorporates multiple novel design features essential for clinical translation, with a focus on scalability, mechanism of delivery, fixation to target tissue, and filling/refilling with a therapeutic cargo. We generated a porous reservoir out of a single material, a flexible thermoplastic polymer, capable of delivering cargo via fill lines to target tissues. We used a radiopaque shear thinning hydrogel for reservoir filling, and tested minimally invasive fixation methods comparing laparoscopic tacks with cyanoacrylate bioadhesive. We then validated the Regenervoir design features and deployment systems in three different large animal models with relevance to cardiac, abdominal, and soft tissue pathologies.

The Regenervoir system provides regional delivery of therapeutic cellular cargo to multiple different target tissues. The overall design concept, independent of clinical application, incorporates a refill line which connects to one or multiple loading chambers that house the therapeutic cargo. Each loading chamber has an active surface, consisting of a semipermeable membrane that allows for the therapeutic cargo housed within the chamber to interact with the host environment via direct contact or paracrine mechanisms. The overall Regenervoir concept is then tailored to specific clinical scenarios with intended targeting to three distinct tissue types: intraperitoneal (ip-Regenervoir), intramuscular (im-Regenervoir), and cardiac (c-Regenervoir). All three designs allow a platform than can target the needs of loco-regional delivery in a minimally invasive manner using indication specific cargoes. Each version of Regenervoir was developed for deployment with a unique delivery system as deployment of each configuration has design challenges as they are constrained in different dimensions based on the anatomical location. For each design, ease of deployment, tissue/organ contacting, alignment to current clinical treatment paradigm, and correct orientation of the reservoir were the common rationale design elements. The ip-Regenervoir system is delivered to the intraperitoneal space through a minimally invasive procedure utilizing a percutaneous delivery sheath for targeting of intra-abdominal pathology. In this configuration, we show the importance of understanding deployment and orientation with anatomical relations and also the fixation requirements in a wet space using a currently approved fixative. The im-Regenervoir system is positioned between muscle layers, with securement to the fascia via a minimally invasive scope and trocar system, providing a means for targeting various soft tissues through an endoscopic procedure. In this configuration, we show that space can be created and a reservoir deployed in an atraumatic manner and secured with current fixation tools. Finally, the c-Regenervoir system is delivered through a minimally invasive incision in the chest (minithoracotomy) and wrapped around the left ventricle, providing targeted delivery directly to the epicardium of the heart. This configuration demonstrates anchoring to a dynamic organ, deployment on a curved surface that requires unsheathing and orientation in a limited space (pericardium) while working through a visually limiting mini-thoracotomy.

\section{Results and Discussion}

\subsection{Regenervoir Fabrication}

We utilized a soft, flexible thermoplastic polymer (thermoplastic polyurethane, or TPU) for the production of the entire reservoir frame (Figure 1a). The use of TPU allowed us to incorporate a variety of manufacturing elements into the Regenervoir housing, such that a single material was manipulated to provide a broad range of design features. The loading chambers were generated using a vacuum thermal former and a custom 3D printed mold. Size, positioning, and orientation were modified depending on the clinical application. An outer channel was added around the loading chamber by heat sealing two layers of TPU around water soluble paper which was subsequently washed out. This enabled fabrication of a separate channel from the loading chamber, for remote delivery of an adhesive for fixation. Internal struts were added to the loading channel with an additional heat sealing phase, in which TPU layers were focally bonded over a 3D printed mold in a heat transfer machine. Fill or purge lines were secured to the Regenervoir system by heat bonding TPU catheter tubing directly to excess material surrounding the loading chamber.

The active surface of Regenervoir consists of a porous layer of TPU that facilitates the therapeutic effect of cellular cargo. 
a)

Fabrication

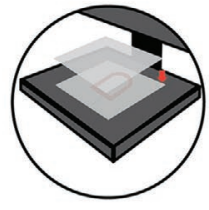

01

Place adhesive channel stencil in between 2 layers of TPU

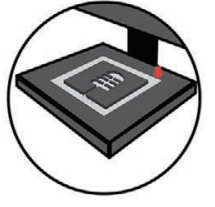

02

Add reservoir mould

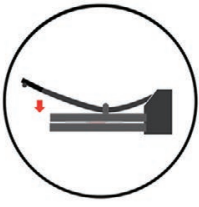

03

Heat Layers in heat press

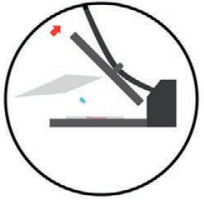

04

Remove from heat press

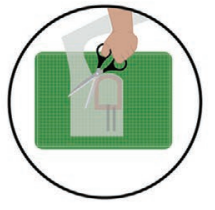

05

Trim excess TPU

from the perimeter of the device

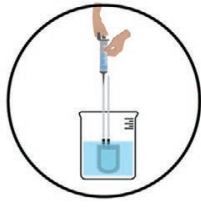

06

Testing \&

quality control

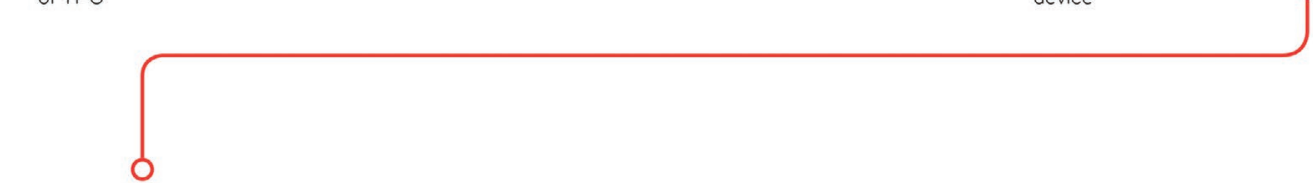

\section{Regenervoir}

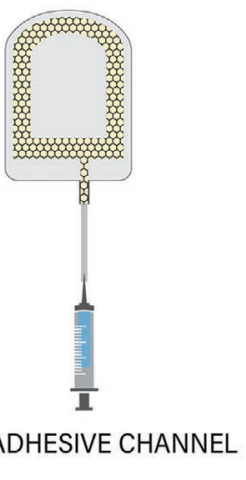

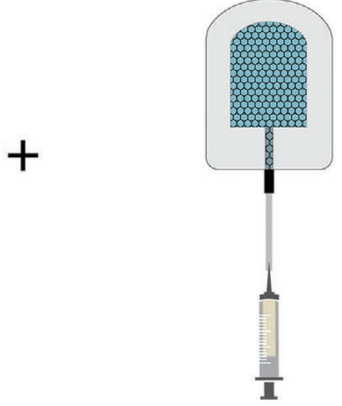

THERAPY CHANNEL

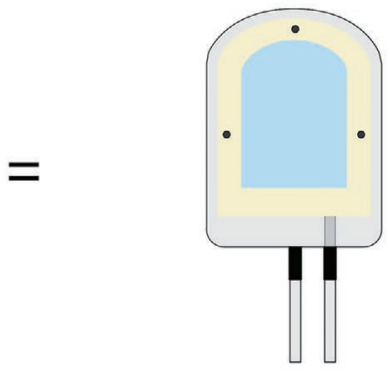

COMPLETED DEVICE
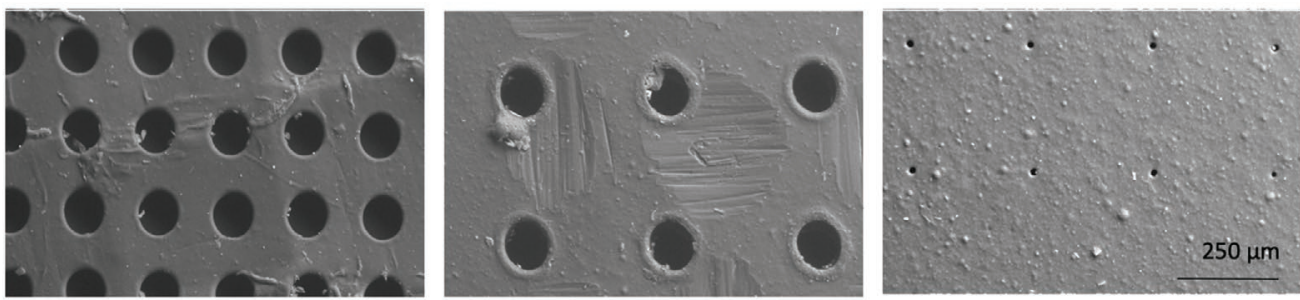

Figure 1. Fabrication of porous reservoirs a) Fabrication of Regenervoir; thermoforming with 3D printed molds enables precise reservoir dimensions with different fill patterns. Heat sealing over 3D printed molds enables 2D patterning of flat reservoirs. Heat Sealing with water soluble paper allows layered overlapping compartments for different channels (adhesive and therapy channels). b) Laser cutting provides controlled porosity at multiple sizes and densities (Left to right: High density $100 \mu \mathrm{m}$ pores, low density $100 \mu \mathrm{m}$ pores, $10 \mu \mathrm{m}$ pores).

A laser cutting technique was used to generate pores from 10 to $100 \mu \mathrm{m}$ in diameter. Pore size, pattern, and density can be varied depending on the specific clinical need, with larger pores allowing migration of the cellular cargo outside of the loading chamber, while pores $<10 \mu \mathrm{m}$ provide an immune-protective barrier (via size exclusion of immune cells) but still allow diffusion of macromolecules and paracrine factors (Figure 1b). We do not claim complete immune isolation with this pore size, but the design is modular to allow the incorporation of membranes with smaller pore size as previously described, ${ }^{[33]}$ if necessary.
The use of TPU as the chamber support material also allowed us to incorporate novel design features into the device that permit delivery, filling, and fixation of the Regenervoir system. With the addition of TPU tubing, we are able to generate the entire implant from a single material, one with a favorable biocompatibility profile when loaded with cells and implanted in rodents. ${ }^{[3,34]}$ The flexible nature of the completed devices also enables them to be used in low profile delivery systems, such that a minimally invasive procedure can be utilized for implantation.

The fill line enables repeated loading of therapeutic cells within the chamber. To preserve the viability of cells during 
a)

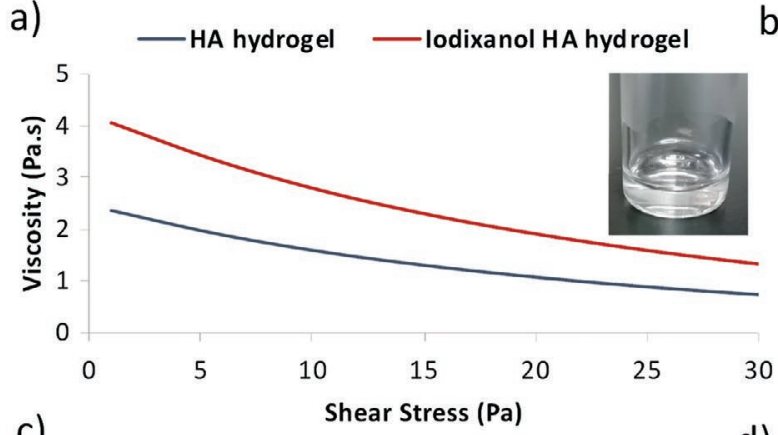

c)

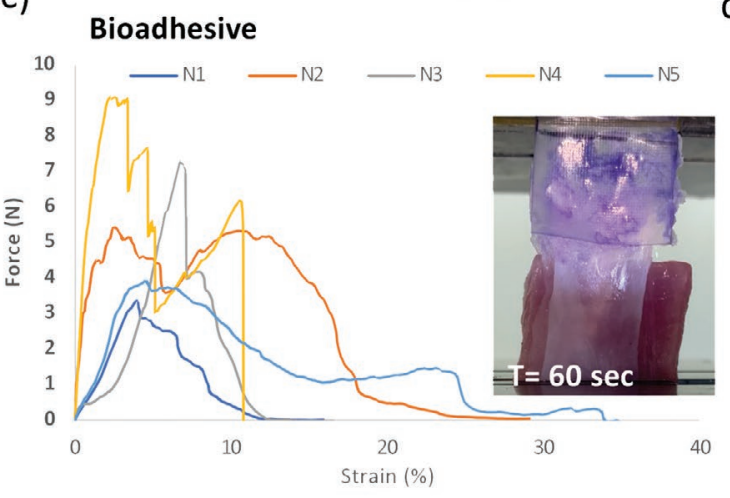

e)

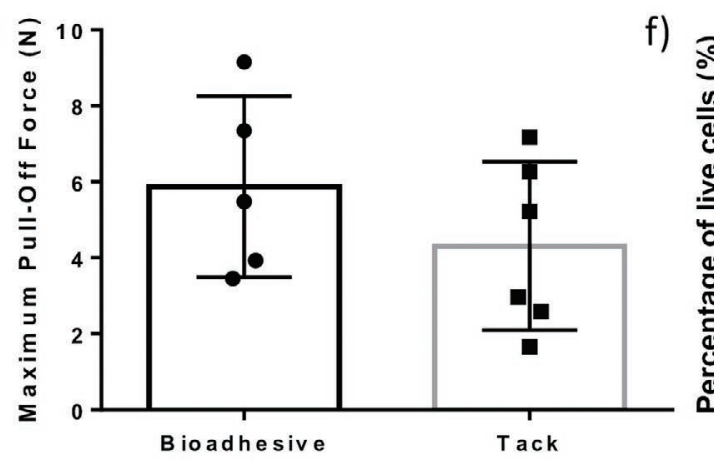

d)

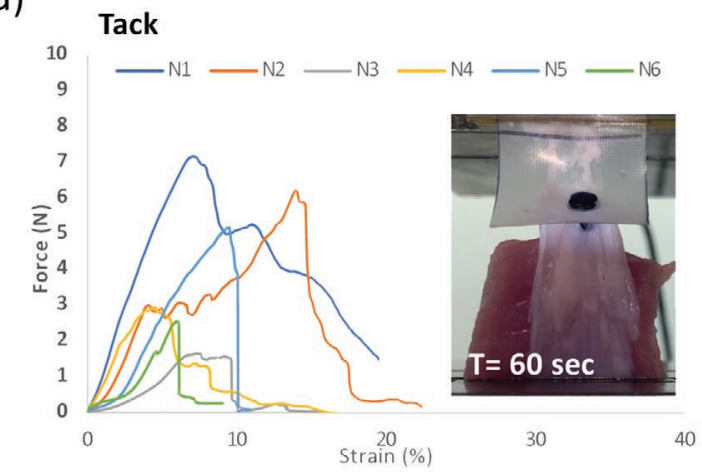

b)

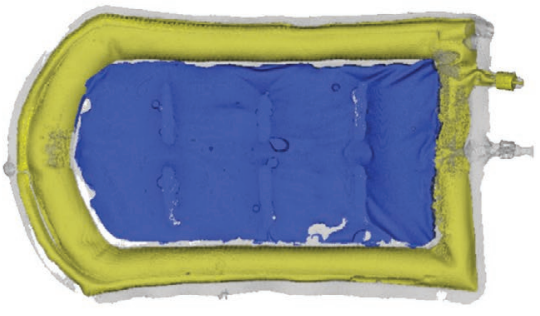

Tack

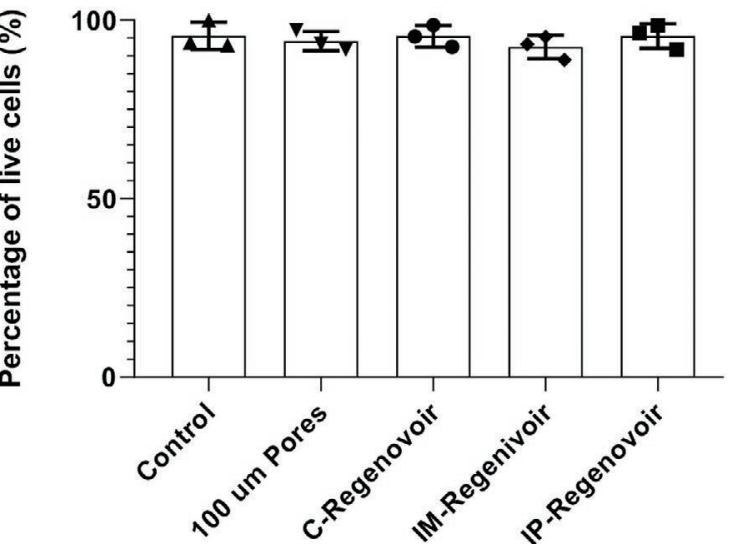

Figure 2. Filling and adhesion of the porous reservoirs. a) Viscosity in response to increase shear stress of HA $1 \% \mathrm{w} / \mathrm{v}$ hydrogel and lodixanol $11.85 \% \mathrm{w} / \mathrm{w} /$ HA $1.1 \% \mathrm{w} / \mathrm{v}$ hydrogel. lodixanol hydrogel and HA 1\%w/v show comparable shear thinning (reduction in viscosity) on application of stress. Data shown representative of the norm $(n=3)$. Inset radiopaque hydrogel is a clear colorless liquid. b) microCT of filled device with reservoir (blue) and adhesive channel (yellow). c) Force versus strain (\%) curve showing adhesive behavior of bioadhesive. d) Force versus strain (\%) curve showing adhesive behavior of tissue tasks. e) Maximum pull-off forces for bioadhesive compared to tissue tacks ( $n=5-6 /$ group, $p=0.25$ ). $\mathrm{f}$ ) $\%$ viability of hMSCs after delivery through $100 \mu \mathrm{m}$ pores and to each configuration of Regenervoir compared to delivery through a standard syringe with an $18 \mathrm{~g}$ needle (control).

infusion and after delivery to the loading chamber, we developed a biomaterial carrier to provide a support matrix that not only enhances cell viability, but also has favorable rheological properties that enable infusion through small bore tubing. Iodixanol HA hydrogel, a hyaluronic acid (HA)-based shearthinning hydrogel incorporating a commercially available Iodixanol contrast agent, was used in this study as a therapeutic cargo analog that allows visualization during deployment. The shear thinning nature of the hydrogel facilitates delivery to the device. While the presence of the Iodixanol in the HA gel results in an increase in resting viscosity it has a comparable rheological profile in terms of shear thinning to HA hydrogel alone as shown in Figure 2a, making it a valuable demonstrator material. We have previously reported cell viability in a crosslinkable version of this HA hydrogel. ${ }^{[35,36]}$ MicroCT imaging of a device with reservoirs filled with different concentrations of Iodixanol HA hydrogel was performed which enabled us to generate a 3D model of the filled device highlighting the different compartments (Figure 2b).

In order to secure the Regenervoir system to underlying tissue, we tested two different fixation methods compatible with existing clinical procedures. First, a medical grade cyanoacrylate 
bioadhesive was administered through a separate fill line with attachment points around the periphery of the device. This is illustrated in the 3D imaging shown in Figure 2b, with reservoir for therapeutic cargo shown in blue and the adhesive channel shown in yellow. For endoscopic fixation, the device was secured with a commercially available laparoscopic tacking device. We then compared adhesion force of the bioadhesive and the tissue tacks adhered to freshly harvested porcine muscle tissue, which was recorded using a lap shear pull-off test set-up. No significant difference was observed between the tissue fixation modalities, tissue tack compared to bioadhesive $(4.31 \pm 2.22$ vs $5.82 \pm 2.38 \mathrm{~N}, p=0.25)$, indicating both techniques are suitable for in vivo use. The mode of failure was noted to be slightly different between the two methods, likely due to the fact that the bioadhesive acts on a larger surface area of muscle tissue (Figure 2c-e).

To demonstrate feasibility of cell delivery, we delivered $33 \times 10^{4}$ human mesenchymal stem cells (hMSCs) $\mathrm{mL}^{-1}$ to each configuration of Regenervoir and through devices with $100 \mu \mathrm{m}$ pores and determined the percentage viability compared to delivery through a standard syringe with an $18 \mathrm{~g}$ needle (control). We found $>90 \%$ viability with no significant differences between the groups (Figure 2f) showing feasibility of cell delivery to the Regenervoir devices and also through the $100 \mu \mathrm{m}$ pores.

\subsection{Validation of Regenervoir Concept in Large Animal Models}

\subsection{1. ip-Regenervoir}

We first sought to establish the intraperitoneal space as a tissue target using a procedure translatable to humans with the ipRegenervoir system. The ip-Regenervoir device consists of an inner loading chamber that can be infused with therapeutic cells, and an outer bio adhesive channel (Figure 3a) which can be filled with a bio adhesive to secure the loading chamber to surrounding tissues. Prior to proceeding with in vivo testing, we confirmed device functionality with benchtop testing. Filling the loading chamber and bioadhesive channel with aqueous dye solutions highlights the separate filling of the different channels (Figure 3a; and Movie S1, Supporting Information).

The ip-Regenervoir and delivery system were designed to be compatible with the skillset of clinicians who perform imageguided procedures (e.g., interventional radiologists), ensuring that the technology is easily adapted for clinical use. A percutaneous approach is an established method for accessing the ip space for tissue biopsies and drain placement. ${ }^{[37]}$ Therefore, we devised a percutaneous, over-the-wire approach to position the ip-Regenervoir in the abdominal cavity (Figure 3b) based on the Seldinger technique. ${ }^{[38]}$ The greater omentum is a highly vascularized bed of adipose and connective tissue that supports the blood supply to the mesentery. It serves as an ideal anatomic target for device delivery, as it offers direct access to the ip space and has been used to support pancreatic islet implants for treatment of diabetes. ${ }^{[39,40]}$ The greater omentum also presents a low risk intra-abdominal implant site, as complications such as intestinal perforation or bowel obstruction are rare, and a free flap of omentum is often mobilized for wound coverage or protection of implanted prosthetic. ${ }^{[38]}$ In swine the greater omentum consists of a highly vascular thin layer of tissue that is adherent to the anterior wall of the stomach, which serves as a useful landmark to guide device positioning. Wire access to the ip space was obtained using ultrasound guided placement of a $16 \mathrm{~g}$ needle. The wire was then positioned in the appropriate intra-abdominal compartment using a combination of fluoroscopic and ultrasound guidance (Figure $3 \mathrm{c}, \mathrm{d}$ ). The device was then loaded into a 30 Fr sheath and deployed over the omentum and oriented with the active surface directed to the posterior surface of the animal. The orientation of the active surface was confirmed with a radiopaque c-shaped marker at the distal end of the device (Figure 3e) and wires and sheaths were removed. A syringe was then used to inject solution into the bioadhesive channel. Cyanoacrylate has been used extensively in surgical procedures for tissue adhesion ${ }^{[41]}$ and embolization $^{[42]}$ and specifically has been used to secure synthetic meshes to the abdominal wall during hernia repair. ${ }^{[43]}$ We therefore utilized a medical grade cyanoacrylate for fixation, and the device was allowed to bond to surrounding tissue for $5 \mathrm{~min}$. Once we confirmed that the device was positioned appropriately, the animal was euthanized, and a CT scan was performed of the animal. The ip-Regenervoir was identified in a 3D rendering of the intact specimen, and cross-sectional imaging confirmed appropriate placement of the device (Figure 3f,g). Postmortem dissection confirmed that the device was in the appropriate place, and the cyanoacrylate was adherent to the omental tissue (Figure 3h).

\subsection{2. im-Regenervoir}

The im-Regenervoir system was designed for cellular delivery to intramuscular targets, using a minimally invasive delivery system congruent with current surgical techniques. Endoscopic techniques are widely used for the repair of abdominal wall defects, such as inguinal hernias ${ }^{[44,45]}$ and ventral hernias. ${ }^{[46]}$ During these procedures, a potential space between muscle layers is accessed and expanded to accommodate a prosthetic implant (typically a hernia mesh) or to facilitate primary repair of the abdominal wall defect. The fascial plane between the internal and external oblique muscles of the anterior abdominal wall is a representative intramuscular implant site as it is easily accessible in the pig, and similar in size to that of humans. Therefore, we demonstrated the clinical validity of the imRegenervoir, through delivery and deployment of the device to the abdominal wall of a pig, and in doing so exploited existing surgical techniques for abdominal wall reconstruction. The loading chamber consists of a continuous square wave pattern with fill and purge lines (Figure 4a; and Movie S2, Supporting Information). The loading chamber pattern was selected to maximize surface area of the active surface of the device. An endoscopic technique was used for device delivery (Figure 4b). The fascial plane between the muscles was first separated using a space creating balloon, then expanded with $\mathrm{CO}_{2}$ insufflation. A single visualization port and two working ports were placed in the potential space, and the im-Regenervoir was rolled so that it could be compressed into a $10 \mathrm{~mm}$ laparoscopic trocar. The im-Regenervoir was then delivered into the insufflated space, 
a)
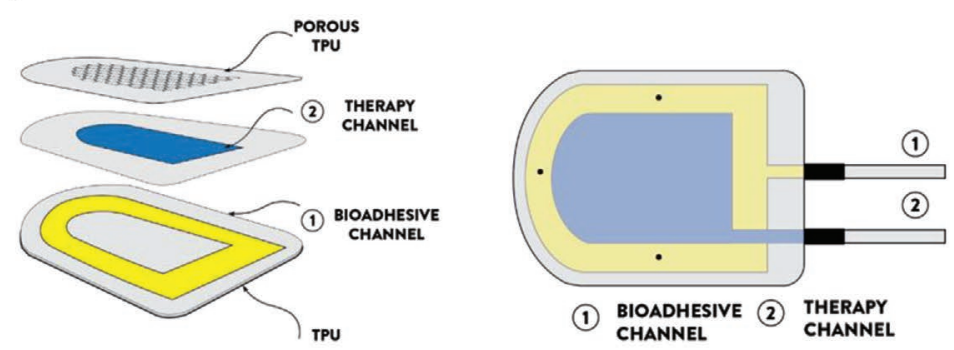

(1) BIOADHESIVE (2) THERAPY
CHANNEL

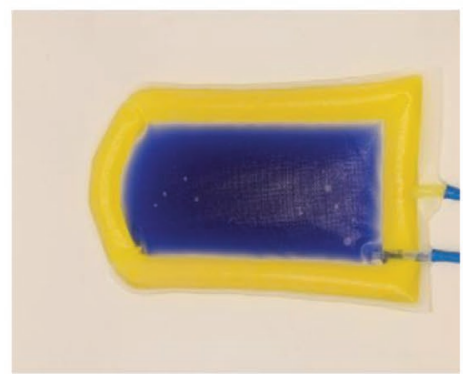

b)

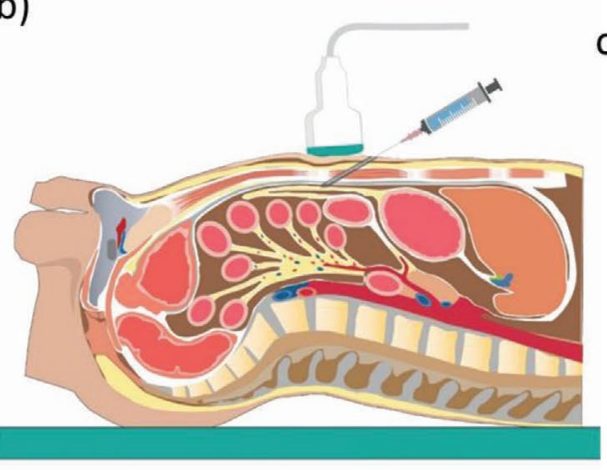

c)

d)
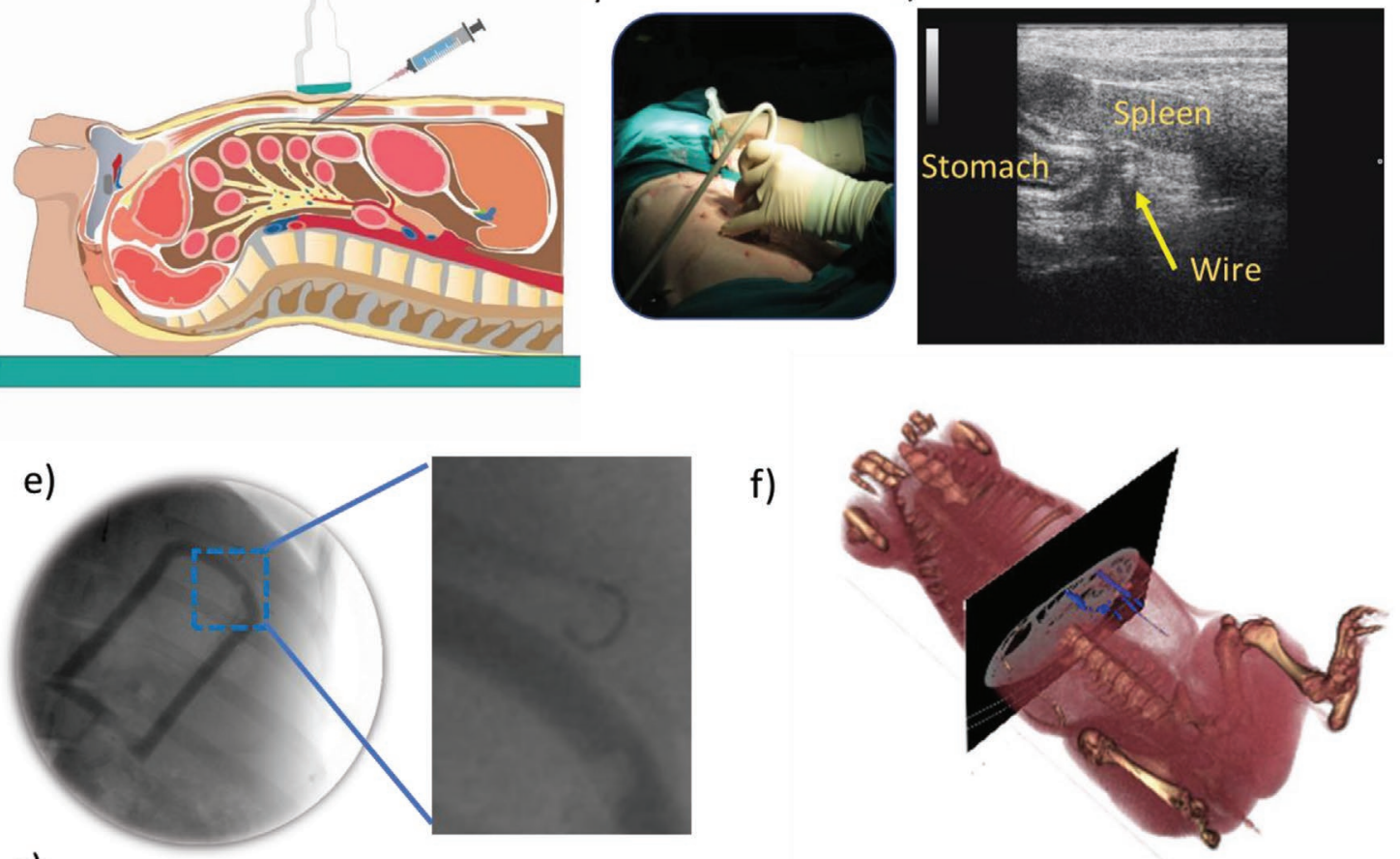

f)

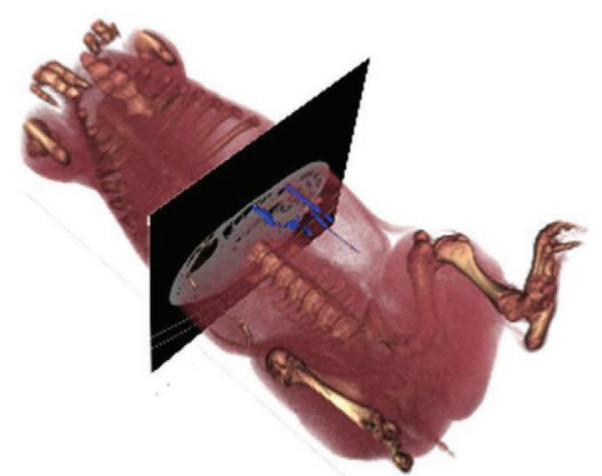

g)
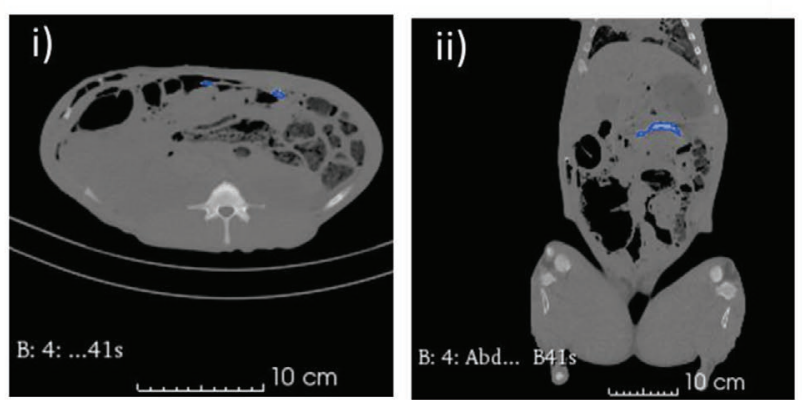

h)

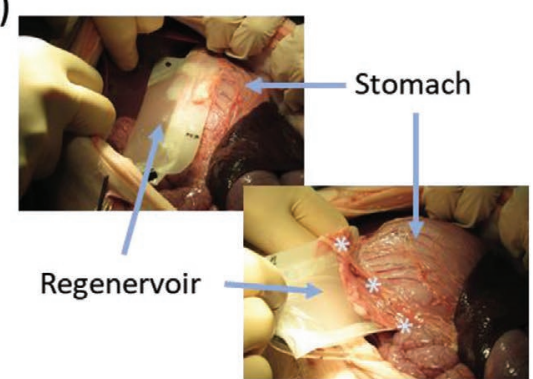

Figure 3. Percutaneous delivery of ip-Regenervoir to the omentum. a) Rendering and produced unit of reservoir system with adhesive (yellow) and therapeutic (blue) channels filling. b) Schematic of procedure for percutaneous delivery to the intraperitoneal space. c) Micrograph of ultrasound guided needle access. d) Ultrasound guided access of the left upper quandrant is used for positioning of device on omentum. A guidewire is used to position a delivery sheath in the tissue plane of the omental fat. e) Fluoroscopy enables visualization of the adhesive channel (filled with contrast here) after implantation, with a distal radiopaque marker to guide orientation of the reservoir. f) 3D rendering of a computed tomography scan of a pig after percutaneous delivery of the reservoir system confirms intra-abdominal positioning. g) A deployed device (blue) is seen on both axial (i) and coronal (ii) slices. h) After infusion of the adhesive channel with cyanoacrylate, the device can be seen fixed to the greater omentum, which overlies the stomach (

opened and positioned under direct visualization. The edges of the device were secured to the underlying internal oblique fascia using a laparoscopic tacking device (Figure 4c), and radiopaque contrast was injected into the fill line under fluoroscopic guidance to verify channel patency (Figure 4d). Postmortem exam confirmed proper placement of the device after the procedure. 

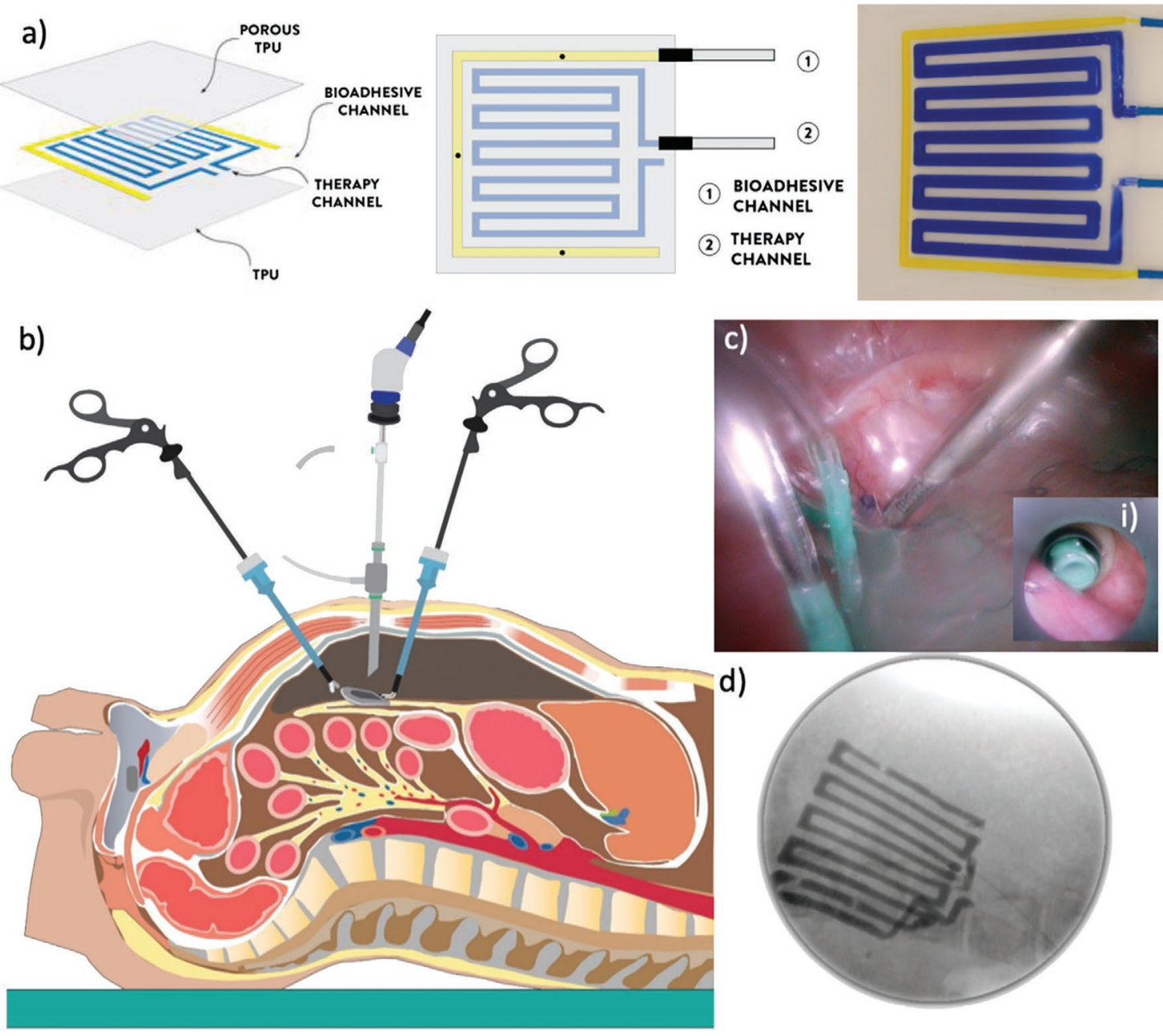

Figure 4. Laparoscopic delivery of im-Regenervoir to the omentum. a) Rendering and physical prototype of im-Regenervoir system with adhesive (yellow) and therapeutic (blue) channels. b) Schematic of the procedure for laparoscopic delivery to the intramuscular space. c) Fixation of the imRegenervoir using a laparoscopic tacking device with tack position confirmed (i). d) Filling of the therapy channel under fluoroscopy.

\subsection{1. c-Regenervoir}

Congestive heart failure, characterized by poorly functioning myocardium as a result of prior myocardial ischemia or congenital abnormalities, is a growing burden on healthcare systems worldwide. ${ }^{[4,48]}$ As treatment paradigms evolve to include cell and biomaterial-based therapies to improve heart function, novel delivery strategies are necessary to deliver these treatments. ${ }^{[2]}$ Although alternative strategies have been devised for delivery of biomaterials and cells to the heart in minimally invasive fashion, including a biodegradable polymer reservoir, ${ }^{[35]}$ a hydrogel patch, ${ }^{[49]}$ and a flexible injection catheter, ${ }^{[50]}$ a refillable depot that can be secured to the heart has not yet been established. The c-Regenervoir system aims to address this unmet need, by creating a multidepot reservoir that wraps around the left ventricle to secure focal delivery of therapeutic cargo. The c-Regenervoir consists of nine loading chambers linked in series with a fill line to allow loading of the device (Figure 5a). Infusion with an aqueous dye shows equitable filling of all loading chambers. The exact number and positioning of loading chambers can be modified according to the specific patient needs. If a patient is suffering primarily from an infarct of the intraventricular septum, the layout of chambers can be modified so that the impact of cargo is centered on the intraventricular septum. A separate channel for bioadhesive was also included to permit fixation to tissue (Figure 5a; and Movie S2, Supporting Information).

The cardiac configuration of Regenervoir was designed to be deployed through a sheath placed through a small intercostal incision (mini-thoracotomy), Figure 5b. To enable precise delivery of the multichambered reservoir, we designed a minimally invasive delivery system to wrap c-Regenervoir around the heart. A shape-memory nitinol frame was developed that molds the Regenervoir system to the silhouette of the underlying ventricle (Figure 5c). A soft vacuum gripper temporarily adheres to the apex of the heart to enable stabilization of the dynamic organ during device delivery and placement. The c-Regenervoir is advanced out of a delivery sheath with the shape-memory nitinol frame expanding to its ventricular confirmation, enabling precise guidance and placement of the reservoir around the heart (Figure 5d). Once the reservoir is adhered 

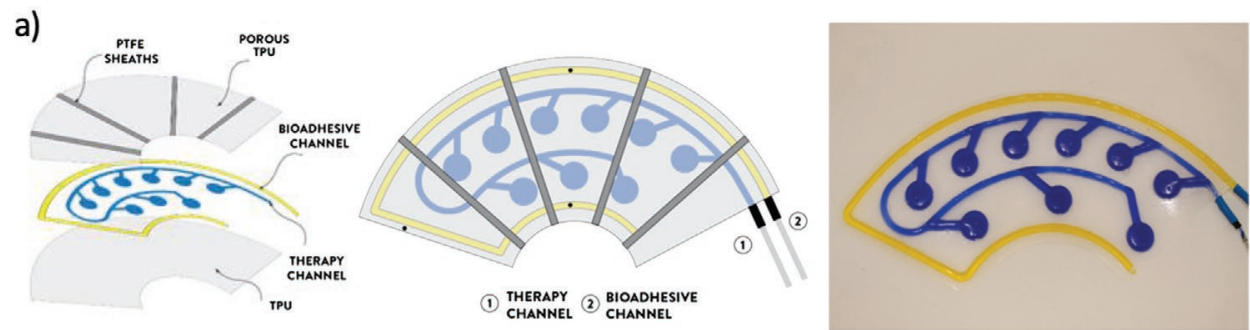

b)

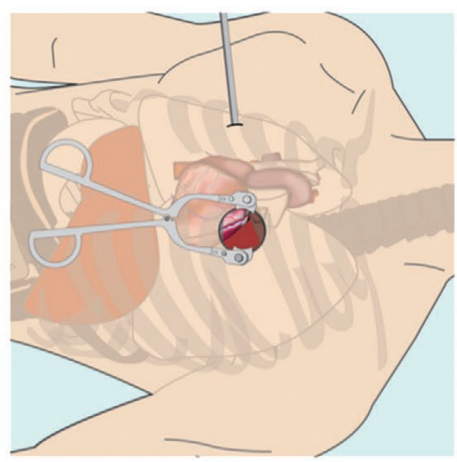

d)

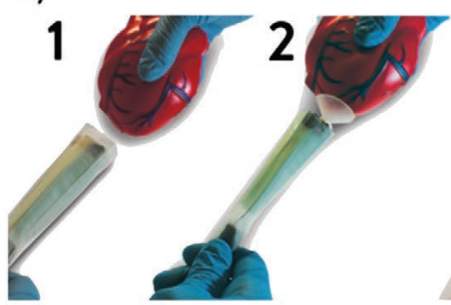

c)

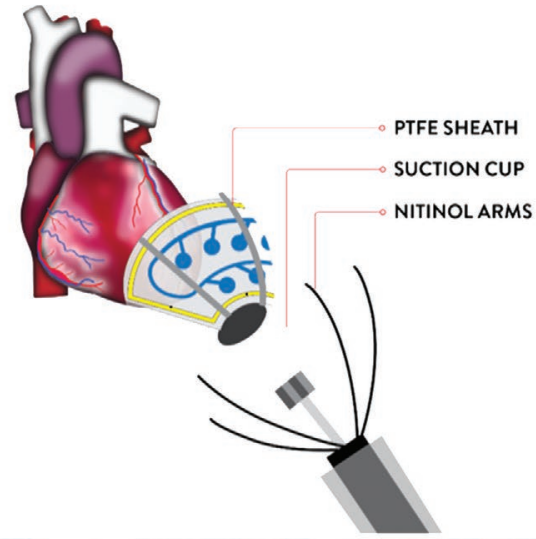

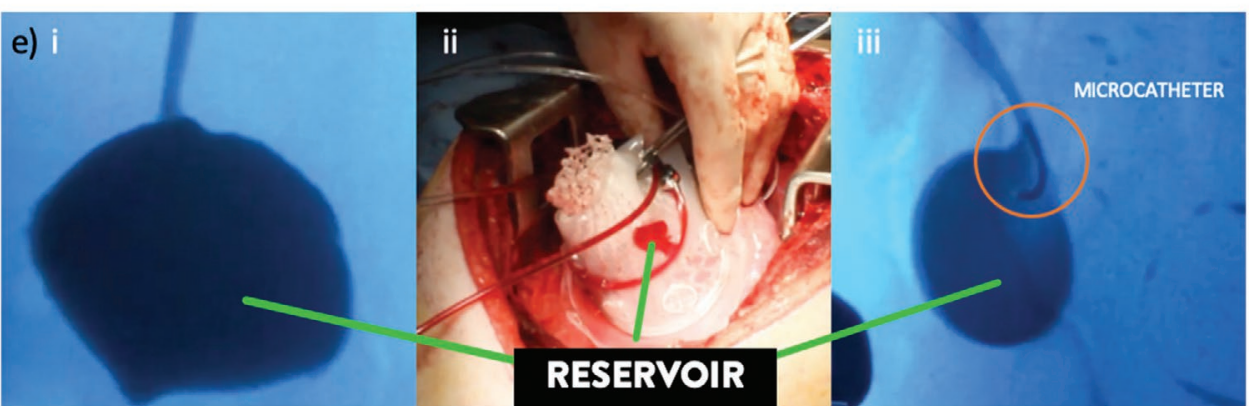

Figure 5. Epicardial delivery of a Regenervoir to the heart. a) Rendering of reservoir system with multiple depots for wrapping around the left ventricle. b) Schematic of the mini-thoracotomy procedure. c) Schematic of positioning and deployment of the reservoir on a moving heart including suction and polytetraflueoroethylene (PTFE) guidance arms. d) Steps for positing and deploying the cardiac reservoir. e) Filling of the cardiac reservoir on the heart during an open chest procedure including fluoroscopy and photographic images of global reservoir filling (i,ii) and a fluoroscopic image of targeted reservoir filling by a microcatheter (iii).

to the epicardial surface, the nitinol frame is withdrawn back into the sheath, leaving the c-Regenervoir fixed in place.

We then tested delivery of the c-Regenervoir system under direct and fluoroscopic visualization by applying it to the epicardial surface via a median sternotomy in a swine model. We show filling of the reservoirs through the fill line with contrast agent (Figure 5e(i)) and dye (Figure 5e(ii)). Finally, we show that the fill lines can be used as a conduit for tracking microcatheters to specific reservoirs for more targeted delivery (Figure 5e(ii)) and we envisage this as a potential biopsy approach if required.

\section{Conclusion}

As the multitude of disease targets that can be treated with cell therapy continues to expand, new technologies are needed to augment the therapeutic potential of these cells through regional and repeated delivery to select tissues. Here we describe a flexible monomaterial polymeric reservoir that can be used to enhance the efficacy of cell therapies in diverse clinical scenarios. We can scale this reservoir to large animal models, while still allowing low profile, minimally invasive delivery, aligned to common interventional procedures. We show predictable deployment of 
the reservoirs at the target site using channels embedded in the reservoirs, a strategy that is easily translated to human studies. Choosing a single thermoplastic materiel to fabricate the reservoir structure allowed us to incorporate novel design features to achieve fixation and filling of the Regenervoir system in three distinct settings. Further, the ability to laser cut the material enables facile porosity tuning to facilitate the unique cell delivery requirements associated with a multitude of disease targets (e.g., cell migration, cell isolation, and cell immune-isolation). For clinical translation, we envisage the Regenervoir enabling multiple discrete therapy refills through injection into a subcutaneous port, or alternatively, connecting the therapy line to a controlled delivery infusion pump, such as those currently used in the clinic for insulin delivery, for continuous therapy infusion. In summary, we feel that this versatile platform presents a significant enhancement over current reservoir platforms for cell/drug delivery due to the multifunctionality (minimally invasive delivery, in situ deployment, adhesion, and tunable therapy delivery) enabled by a functionalized monomaterial reservoir.

\section{Experimental Section}

Fabrication of Reservoir System: The manufacturing technique for the Regenervoir systems is shown in Figure $1.0 .3 \mathrm{~mm}$ thick TPU sheets for the loading chamber and $0.075 \mathrm{~mm}$ TPU sheets for the membrane and channels (HTM-8001-M and HTM-1001 polyether TPU film, American Polyfilm, Inc) were utilized. The loading chambers were formed into desired shapes based on a 3D printed mold for each prototype (intraperitoneal, intramuscular, or cardiac). 3D molds were designed with Autodesk Inventor and printed with a Formlabs2 printer. The loading chamber was shaped using a vacuum thermal former (450DT, Formech). To incorporate a bioadhesive channel, a section of water soluble paper (2301S Print-Stitch-Dissolve, Pellon) was secured between two $0.075 \mathrm{~mm}$ TPU sheets which were then heat bonded using a heat transfer machine (Heat Transfer Machine QXAi, Powerpress). Perforations to allow for diffusion of bioadhesive were made using a 21 gauge needle. The fill lines were added using thermoplastic polyurethane catheter tubing (ID $=1 \mathrm{~mm}, \mathrm{OD}=1.97 \mathrm{~mm}$, MRE 080 Micro-Renathane, Braintree scientific) which was bonded to this assembly using a heat transfer machine. This reservoir interfaces with soft tissue via the TPU membrane which can have pores of variable size. 10-100 $\mu \mathrm{m}$ pores (National Centre for Laser Applications, National University of Ireland Galway) were generated in the TPU membrane.

Fabrication of c-Regenervoir Delivery System: To create the nitinol frame, $0.5 \mathrm{~mm}$ superelastic Nitinol wire (Nexmetal Corp) was fixed around a ventricular shaped stainless-steel mold and annealed in a furnace at $500{ }^{\circ} \mathrm{C}$ for $30 \mathrm{~min}$. The shape-set nitinol frame was then secured around the end of a catheter ( $4.5 \mathrm{~mm}$ ID, $6 \mathrm{~mm}$ OD). The soft, silicone suction cup gripper (Ecoflex 00-20) was attached to the end of a vacuum line $(3 \mathrm{~mm} I D, 4 \mathrm{~mm}$ OD) and placed within the inner lumen of the catheter. The c-Regenervoir was fabricated as described previously, with the addition of four TPU sleeves on the back of the reservoir. PTFE sheaths ( $2 \mathrm{~mm} \mathrm{ID,} 3 \mathrm{~mm}$ OD, Allen Tech) were inserted into each of the four sleeves to serve as guides for the nitinol arms and to reduce friction between the device and the nitinol arms during device deployment. The c-Regenervoir was loaded onto the nitinol frame, with the four nitinol arms sliding into the PTFE sheaths. The entire system was then loaded within a delivery sheath $(9 \mathrm{~mm} I D$, $13 \mathrm{~mm}$ OD) and was ready for use. After guiding the delivery sheath through the intercostal space and into the thoracic cavity, the surgeon can move the suction cup gripper and the nitinol frame independently within the sheath for optimal device placement and deployment.

Production of Shear Thinning Hydrogel: A HA-based hydrogel incorporating a contrast agent was used in this study. The hydrogel, herein referred to as lodixanol HA hydrogel, was required to have a shear thinning profile which facilitates injectability. The HA base hydrogel was prepared using a long chain, high molecular weight ( $\approx 1.1-1.3 \mathrm{MDa}) \mathrm{HA}$ polymer with no side chain substitution at a concentration of either $1 \%$ or $1.447 \% \mathrm{w} / \mathrm{v}$ (for lodixanol hydrogel) in purified water. lodixanol (Visipaque $320 \mathrm{mg}$ lodine $\mathrm{mL}^{-1}$, GE Healthcare) is a commercially available iodinated contrast agent. Incorporation into a hydrogel at a concentration of $9.22 \% \mathrm{w} / \mathrm{w}$ to create a radio opaque formulation is previously documented. ${ }^{[5]}$ For these studies, the lodixanol HA hydrogel was prepared in aliquots $(11 \mathrm{~mL})$ using a combination of $1.447 \% \mathrm{w} / \mathrm{v}$ $\mathrm{HA}$ hydrogel $(9 \mathrm{~mL})$ and lodixanol $(2 \mathrm{~mL})$. These were mixed until homogeneous giving an lodixanol $\mathrm{HA}$ gel at a final concentration of $1.2 \% \mathrm{w} / \mathrm{v} \mathrm{HA}$ and $11.85 \% \mathrm{w} / \mathrm{w}$ lodixanol. Rheological properties were assessed using a Discovery Hybrid Rheometer HR-2 (TA instruments, USA). Viscosity of each hydrogel was measured by flow rheometry using cone and plate (40 mm cone geometry with $4^{\circ}$ angle). All analysis was performed at a constant $25{ }^{\circ} \mathrm{C}$ with controlled shear stress applied across the range 1-30 $\mathrm{Pa}$. All hydrogels were measured in triplicate and rheograms shown (Figure $2 \mathrm{f}$ ) are representative of the norm.

Mechanical Testing of Tissue Fixation Modalities: A Zwick mechanical testing machine (Z050, Zwick/Roell) with a $100 \mathrm{~N}$ load cell was used for pull-off testing to determine the adhesion forces of the tissue fixation modalities. Freshly harvested porcine abdominal muscle was cut into $3 \times 2 \times 0.5 \mathrm{~cm}$ samples. $3 \times 2 \mathrm{~cm}$ samples of TPU were prepared and $1.5 \mathrm{~cm}$ of TPU was placed on top of the tissue. A single Poly-d, I-Lactic Acid (PDLLA) resorbable laparoscopic tack (Bard) was inserted into the center of the sample using the accompanying tack applier. The tissue cyanoacrylate (Histoacryl, B. Braun) was prepared following manufacturers protocol and tissue cyanoacrylate $(100 \mu \mathrm{L})$ was injected onto the tissue before the TPU samples were placed on top, where $1.5 \mathrm{~cm}$ of the TPU was glued to the tissue. The samples were mounted in the tensile tester using pneumatic clamps at $6 \mathrm{PSI}$, tests were ran at a shear rate of $20 \mathrm{~mm} \mathrm{~min}^{-1}{ }^{[52]}$ and maximum lap shear force was recorded ( $n=5-6$ per group).

In Vitro Cell Viability: hMSCs were isolated from bone marrow aspirates obtained from human volunteers, at REMEDI, the National University of Ireland Galway. All procedures were carried out with informed consent and ethically approved by the Clinical Research Ethical Committee at University College Hospital, Galway. hMSCs were isolated using standard protocols and stringent analysis of cell phenotype as previously described. [53] hMSCs were cultured using low glucose Dulbecco's modified Eagle's medium (D6046, Sigma) supplemented with $10 \%$ fetal bovine serum, $100 \mathrm{U} \mathrm{mL}^{-1}$ penicillin, $100 \mu \mathrm{g} \mathrm{mL}^{-1}$ streptomycin (Sigma-Aldrich, Ireland), and $5 \mathrm{ng} \mathrm{mL}^{-1}$ bFGF (R\&D Systems) at $37^{\circ} \mathrm{C}$ in a $5 \%$ carbon dioxide $\left(\mathrm{CO}_{2}\right)$ environment. Cells were passaged at 80-90\% confluency, and were not used beyond passage 5. hMSCs were trypsinized, counted, and suspended in the media at a density of $33 \times 10^{4}$ cells $\mathrm{mL}^{-1}$ before loading into a $10 \mathrm{~mL}$ standard syringe with an $18 \mathrm{~g}$ needle. Solution was slowly injected into each configuration of Regenervoir, through devices with $100 \mu \mathrm{m}$ pores and compared to cell injection through the syringe and $18 \mathrm{~g}$ needle alone. The hMSC cell suspension was then diluted with Trypan blue. The assay was based on the permeable nature of the cell membrane in nonviable cells, whereby nonviable cells were stained blue on the addition of Trypan Blue solution, whereas viable cells excluded the dye and remain unstained. The cell concentration and viability were calculated by 2 blinded counters.

Animal Studies: Large animal testing of the ip-Regenervoir and im-Regenervoir devices were approved by the Italian Ministry of Health (No. 976/2017-PR) and performed at Explora Biotech Srl (Italy). Prior to the experiments, the animals were housed in single cages and were subjected to a 1 week acclimatization period following Directive 2010/63/ EU. Prior to surgery, animals received $10 \mathrm{mg} \mathrm{kg}^{-1}$ ketamine (KetaVet 100. MSD, Rome, Italy), $0.5 \mathrm{mg} \mathrm{kg}^{-1}$ diazepam (Hospira, Naples, Italy). Anesthesia was induced by $5 \mathrm{mg} \mathrm{kg}^{-1}$ ketamine and $0.5 \mathrm{mg} \mathrm{kg}^{-1}$ diazepam. After intubation, 2-3\% isoflurane (IsoFlo, Esteve, Rome, Italy) was administrated by mask to maintain anesthesia. Following completion of the acute surgeries, animals were euthanized (IV injection of Tanax, $0.3 \mathrm{~mL} \mathrm{~kg}^{-1}$; MSD Animal Health Srl-Italy), and a postmortem 
dissection was carried out to confirm positioning of the device. The total procedure lasted $6 \mathrm{~h}$, where procedure implant time was $\approx 2 \mathrm{~h}$, with in situ testing and imaging performed in the acute setting.

Intra-Abdominal and Intramuscular Delivery of Regenervoir: Female Landrace pigs, weighting 25-30 kg were utilized for these arms of the study. The ip-Regenervoir was delivered through the intraperitoneal space using an over-the-wire technique $(n=4)$. The peritoneum was entered with an $18 \mathrm{~g}$ needle under direct ultrasound guidance using a $5-13 \mathrm{MHz}$ linear probe (Esaote). A 0.035 in. guidewire was advanced over the greater omentum, and position was confirmed using fluoroscopy (to determine location of gastric bubble) and ultrasound. The delivery tract was dilated using an Amplatz renal dilator set (Boston Scientific), and a custommade $30 \mathrm{Fr}$ sheath was advanced over the omentum. The ip-Regenervoir device was then delivered through the sheath, and positioning was confirmed with fluoroscopy (OEC 9800 Plus, GE HealthCare, Salt Lake City, UT), such that the active surface was directed toward the omentum. A cyanoacrylate adhesive (Histoacryle) was injected into the bioadhesive channel to bond the device to underlying tissue, and the device was filled with the HA/locixanol hydrogel. Following completion of the procedure, animals were euthanized (IV injection of Tanax, $0.3 \mathrm{~mL} \mathrm{~kg}^{-1}$; MSD Animal Health Srl-Italy). A postmortem CT scan (Siemens Syngo CT VC40-16slice) was obtained to confirm positioning of the device, and autopsy was carried out to verify deployment.

The im-Regenervoir system was delivered between muscles the abdominal wall $(n=2)$. A commercially available dissecting balloon and trocar (Spacemaker, Medtronic) was used to gain endoscopic access to the tissue plane posterior to the external oblique muscle, which was insufflated with continuous $\mathrm{CO}_{2}$ in order to maintain the space. Two $5 \mathrm{~mm}$ working ports were placed under direct visualization, and the device was rolled and passed through one of the ports sites to be introduced in the space. The im-Regenervoir device was positioned under direct endoscopic visualization, and secured at the corners using PDLLA laparoscopic tacks (Bard). The device was then filled with the $\mathrm{HA} /$ locixanol hydrogel under fluoroscopic visualization. Following completion of the procedure, animals were euthanized as above and an autopsy was performed to verify deployment.

Cardiac Delivery of c-Regenervoir: Preliminary feasibility of c-Regenervoir was tested on a porcine model. The acute porcine study was performed at the Boston Children's Hospital, and the experimental protocol was approved by the hospital's Institutional Animal Care and Use Committee. 1 female Yorkshire swine (Parsons Farms) with a body weight of $60 \mathrm{~kg}$ was used. Anesthesia, lidocaine delivery, and monitoring were conducted as previously described. ${ }^{[54]}$ Briefly, anesthesia was induced with a mixture of tiletamine/zolazepam $\mathrm{HCl}$ (Telazol, $4.5 \mathrm{mg} \mathrm{kg}^{-1}$, Zoetis), xylazine (2 $\mathrm{mg} \mathrm{kg}^{-1}$, Akorn), and atropine (0.04 $\mathrm{mg} \mathrm{Kg}^{-1}$ intramuscularly, Westward). Isoflurane (0.5-1\%, Baxter) in oxygen was used to maintain anesthesia. The animal was intubated after induction and mechanically ventilated using volume control at $10 \mathrm{~mL} \mathrm{~kg}$ at a rate of $10-20$ breaths $\mathrm{min}^{-1}$. Lidocaine was administered prophylactically to reduce the risk of ventricular arrhythmias caused by the epicardial positioning of c-Regenervoir. A bolus intravenous injection dose of lidocaine ranging from 0.5 to $1.5 \mathrm{mg} \mathrm{kg}^{-1}$ was administered at a rate of $\approx 0.35-0.7 \mathrm{mg} \mathrm{kg}^{-1} \mathrm{~min}^{-1}$. Continuous ECG tracings, arterial blood pressure, and central venous blood pressure were monitored through femoral lines, peripheral capillary, and oxygen saturation percentage. A median sternotomy was used to access the heart. The pericardium was removed and the c-Regenervoir was placed around the heart. The reservoirs were filled with contrast (Omnipaque, global filling or local filling with a microcatheter), while c-arm fluoroscopy (Siemens' AXIOM Artis) was used for imaging and video capture. Dye was delivered to the reservoirs to visualize filling on a beating heart. Euthanization was conducted by an intravenous injection of sodium pentobarbitol (FatalPlus, $110 \mathrm{mg} \mathrm{kg}^{-1}$, Baxter).

\section{Supporting Information}

Supporting Information is available from the Wiley Online Library or from the author.

\section{Acknowledgements}

G.P.D., S.T.R., E.T.R., and E.B.D. contributed equally to this work. E.T.R. acknowledges funding from the Mechanical Engineering Department and the Institute for Medical Engineering and Science at the Massachusetts Institute of Technology. S.T.R. and G.P.D. acknowledge funding from Science Foundation Ireland under Grant No. SFI/12/ RC/2278, Advanced Materials and Bioengineering Research (AMBER) Centre, National University of Ireland and Trinity College Dublin, Ireland. G.P.D. acknowledges financial support from the National University of Ireland Galway. E.B.D., L.M.D., H.K., and G.P.D. acknowledge the DRIVE project which has received funding from the European Union's Horizon 2020 Framework Programme under Grant Agreement No. 645991. S.T.R. has received funding from the European Union's Horizon 2020 research and innovation program under the Marie Skłodowska-Curie Actions Grant Agreement No. 713567. The authors would also like the acknowledge the following grants for use of the hMSCs described in this publication; 09/SRC/B1794 REMEDI grant from Science Foundation Ireland and "PurStem" and FP7 European Union Health Collaborative Project, EU FP7-HEALTH-2007-B, PurStem, Grant No. 223298.

\section{Conflict of Interest}

The authors declare no conflict of interest.

\section{Keywords}

cell and drug delivery, minimally invasive therapeutics, regional and repeated delivery, therapeutic reservoirs

Received: February 21, 2020

Published online: April 27, 2020

[1] M. N. Banerjee, R. Bolli, J. M. Hare, Circ. Res. 2018, 123, 266.

[2] H. S. O'Neill, L. B. Gallagher, J. O'Sullivan, W. Whyte, C. Curley, E. Dolan, A. Hameed, J. O'Dwyer, C. Payne, D. O'Reilly, E. Ruiz-Hernandez, E. T. Roche, F. J. O'Brien, S. A. Cryan, H. Kelly, B. Murphy, G. P. Duffy, Adv. Mater. 2016, 28, 5648.

[3] M.-C. Vantyghem, E. J. P. de Koning, F. Pattou, M. R. Rickels, Lancet 2019, 394, 1274.

[4] B. S. Guerrouahen, H. Sidahmed, A. Al Sulaiti, M. Al Khulaifi, C. Cugno, Stem Cells Int. 2019, 2019, 7219297.

[5] N. Joyce, G. Annett, L. Wirthlin, S. Olson, G. Bauer, J. A. Nolta, Regener. Med. 2010, 5, 933.

[6] J. S. Lunn, S. A. Sakowski, J. Hur, E. L. Feldman, Ann. Neurol. 2011, 70, 353.

[7] R. G. Majzner, C. L. Mackall, Nat. Med. 2019, 25, 1341.

[8] N. Raje, J. Berdeja, Y. Lin, D. Siegel, S. Jagannath, D. Madduri, M. Liedtke, J. Rosenblatt, M. V. Maus, A. Turka, L. P. Lam, R. A. Morgan, K. Friedman, M. Massaro, J. Wang, G. Russotti, Z. Yang, T. Campbell, K. Hege, F. Petrocca, M. Travis Quigley, N. Munshi, J. N. Kochenderfer, N. Engl. J. Med. 2019, 380, 1726.

[9] S. L. Maude, T. W. Laetsch, J. Buechner, S. Rives, M. Boyer, H. Bittencourt, P. Bader, M. R. Verneris, H. E. Stefanski, G. D. Myers, M. Qayed, B. De Moerloose, H. Hiramatsu, K. Schlis, K. L. Davis, P. L. Martin, E. R. Nemecek, G. A. Yanik, C. Peters, A. Baruchel, N. Boissel, F. Mechinaud, A. Balduzzi, J. Krueger, C. H. June, B. L. Levine, P. Wood, T. Taran, M. Leung, K. T. Mueller, Y. Zhang, K. Sen, D. Lebwohl, M. A. Pulsipher, S. A. Grupp, N. Engl. J. Med. 2018, 378, 439.

[10] S. J. Schuster, M. R. Bishop, C. S. Tam, E. K. Waller, P. Borchmann, J. P. McGuirk, U. Jäger, S. Jaglowski, C. Andreadis, J. R. Westin, 
I. Fleury, V. Bachanova, S. R. Foley, P. J. Ho, S. Mielke, J. M. Magenau, H. Holte, S. Pantano, L. B. Pacaud, R. Awasthi, J. Chu, Ö. Anak, G. Salles, R. T. Maziarz, N. Engl. J. Med. 2019, 380, 45.

[11] S. H. Li, T. Y. Y. Lai, Z. Sun, M. Han, E. Moriyama, B. Wilson, S. Fazel, R. D. Weisel, T. Yau, J. C. Wu, R. K. Li, J. Thorac. Cardiovasc. Surg. 2009, 137, 1225.

[12] J. J. Gavira, E. Nasarre, G. Abizanda, M. Pérez-Ilzarbe, A. De Martino-Rodriguez, J. A. García De Jalón, M. Mazo, A. Maclas, I. García-Bolao, B. Pelacho, D. Martínez-Caro, F. Prósper, Eur. Heart J. 2010, 31, 1013.

[13] M. A. Laflamme, S. Zbinden, S. E. Epstein, C. E. Murry, Annu. Rev. Pathol.: Mech. Dis. 2007, 2, 307.

[14] M. Wang, C. Liang, H. Hu, L. Zhou, B. Xu, X. Wang, Y. Han, Y. Nie, S. Jia, J. Liang, K. Wu, Sci. Rep. 2016, 6, 30696.

[15] L. Sánchez, I. Gutierrez-Aranda, G. Ligero, R. Rubio, M. Muñoz-López, J. L. García-Pérez, V. Ramos, P. J. Real, C. Bueno, R. Rodríguez, M. Delgado, P. Menendez, Stem Cells 2011, 29, 251.

[16] Y. Nam, S. M. Jung, Y. A. Rim, H. Jung, K. Lee, N. Park, J. Kim, Y. Jang, Y.-B. Park, S.-H. Park, J. H. Ju, PLoS One 2018, 13, e0198740.

[17] Y. Nam, S. M. Jung, Y. A. Rim, H. Jung, K. Lee, N. Park, J. Kim, Y. Jang, Y. B. Park, S. H. Park, J. H. Ju, PLoS One 2018, 13, e0198740.

[18] S. C. Katz, G. R. Point, M. Cunetta, M. Thorn, P. Guha, N. J. Espat, C. Boutros, N. Hanna, R. P. Junghans, Cancer Gene Ther. 2016, 23, 142.

[19] W. X. Ang, Z. Li, Z. Chi, S. H. Du, C. Chen, J. C. K. Tay, H. C. Toh, J. E. Connolly, X. H. Xu, S. Wang, Oncotarget 2017, 8, 13545.

[20] J. P. Murad, A. K. Kozlowska, H. J. Lee, M. Ramamurthy, W. C. Chang, P. Yazaki, D. Colcher, J. Shively, M. Cristea, S. J. Forman, S. J. Priceman, Front. Immunol. 2018, 9, 2268.

[21] R. J. Powell, W. A. Marston, S. A. Berceli, R. Guzman, T. D. Henry, A. T. Longcore, T. P. Stern, S. Watling, R. L. Bartel, Mol. Ther. 2012, 20, 1280.

[22] D. W. Losordo, M. R. Kibbe, F. Mendelsohn, W. Marston, V. R. Driver, M. Sharafuddin, V. Teodorescu, B. N. Wiechmann, C. Thompson, L. Kraiss, T. Carman, S. Dohad, P. Huang, C. E. Junge, K. Story, T. Weistroffer, T. M. Thorne, M. Millay, J. P. Runyon, R. Schainfeld, Circ.: Cardiovasc. Interventions 2012, 5, 821.

[23] P. Huang, S. Li, M. Han, Z. Xiao, R. Yang, Z. C. Han, Diabetes Care 2005, 28, 2155.

[24] S. Matoba, T. Tatsumi, T. Murohara, T. Imaizumi, Y. Katsuda, M. Ito, Y. Saito, S. Uemura, H. Suzuki, S. Fukumoto, Y. Yamamoto, R. Onodera, S. Teramukai, M. Fukushima, H. Matsubara, Am. Heart J. 2008, 156, 1010.

[25] I. Arutyunyan, T. Fatkhudinov, A. Elchaninov, O. Vasyukova, A. Makarov, N. Usman, E. Kananykhina, A. Lokhonina, D. Goldshtein, G. Bolshakova, G. Sukhikh, Histol. Histopathol. 2019, 34, 513.

[26] N. Sevivas, F. G. Teixeira, R. Portugal, L. Araújo, L. F. Carriço, N. Ferreira, M. Vieira da Silva, J. Espregueira-Mendes, S. Anjo, B. Manadas, N. Sousa, A. J. Salgado, S. C. Serra, Am. J. Sports Med. 2017, 45, 179.

[27] C. J. Chen, F. C. Cheng, H. L. Su, M. L. Sheu, Z. H. Lu, C. Y. Chiang, D. Y. Yang, J. Sheehan, H. C. Pan, PLoS One 2015, 10, e0124624.

[28] E. T. Roche, C. L. Hastings, S. A. Lewin, D. E. Shvartsman, Y. Brudno, N. V. Vasilyev, F. J. O'Brien, C. J. Walsh, G. P. Duffy, D. J. Mooney, Biomaterials 2014, 35, 6850.

[29] Y. Guo, M. Wysoczynski, Y. Nong, A. Tomlin, X. Zhu, A. M. Gumpert, M. Nasr, S. Muthusamy, H. Li, M. Book, A. Khan, K. U. Hong, Q. Li, R. Bolli, Basic Res. Cardiol. 2017, 112, 18.

[30] Y. Tokita, X.-L. Tang, Q. Li, M. Wysoczynski, K. U. Hong, S. Nakamura, W.-J. Wu, W. Xie, D. Li, G. Hunt, Q. Ou, H. Stowers, R. Bolli, Circ. Res. 2016, 119, 635.

[31] X.-L. Tang, S. Nakamura, Q. Li, M. Wysoczynski, A. M. Gumpert, W.-J. Wu, G. Hunt, H. Stowers, Q. Ou, R. Bolli, J. Am. Heart Assoc. 2018, 7, e007400.
[32] M. Wysoczynski, A. Khan, R. Bolli, Circ. Res. 2018, 123, 138.

[33] W. Whyte, E. T. Roche, C. E. Varela, K. Mendez, S. Islam, H. O'Neill, F. Weafer, R. N. Shirazi, J. C. Weaver, N. V. Vasilyev, P. E. McHugh, B. Murphy, G. P. Duffy, C. J. Walsh, D. J. Mooney, Nat. Biomed. Eng. 2018, 2, 416.

[34] E. B. Dolan, C. E. Varela, K. Mendez, W. Whyte, R. E. Levey, S. T. Robinson, E. Maye, J. O'Dwyer, R. Beatty, A. Rothman, Y. Fan, J. Hochstein, S. E. Rothenbucher, R. Wylie, J. R. Starr, M. Monaghan, P. Dockery, G. P. Duffy, E. T. Roche, Sci. Rob. 2019, 4, eaax7043.

[35] E. B. Dolan, B. Hofmann, M. H. de Vaal, G. Bellavia, S. Straino, L. Kovarova, M. Pravda, V. Velebny, D. Daro, N. Braun, D. S. Monahan, R. E. Levey, H. O'Neill, S. Hinderer, R. Greensmith, M. G. Monaghan, K. Schenke-Layland, P. Dockery, B. P. Murphy, H. M. Kelly, S. Wildhirt, G. P. Duffy, Mater. Sci. Eng., C 2019, 103, 109751.

[36] L. B. Gallagher, E. B. Dolan, J. O'Sullivan, R. Levey, B. L. Cavanagh, L. Kovarova, M. Pravda, V. Velebny, T. Farrell, F. J. O'Brien, G. P. Duffy, Acta Biomate. 2020, https://doi.org/10.1016/j. actbio.2020.02.043.

[37] R. F. Dondelinger, Lancet 1999, 353, S15.

[38] S. I. Seldinger, Acta Radiol. 1953, 39, 368.

[39] D. A. Baidal, C. Ricordi, D. M. Berman, A. Alvarez, N. Padilla, G. Ciancio, E. Linetsky, A. Pileggi, R. Alejandro, N. Engl. J. Med. 2017, 376, 1887.

[40] D. M. Berman, R. D. Molano, C. Fotino, U. Ulissi, J. Gimeno, A. J. Mendez, N. M. Kenyon, N. S. Kenyon, D. M. Andrews, C. Ricordi, A. Pileggi, Diabetes 2016, 65, 1350 .

[41] D. García Cerdá, A. M. Ballester, A. Aliena-Valero, A. CarabénRedaño, J. M. Lloris, Surg. Today 2015, 45, 939.

[42] H. Hill, J. F. B. Chick, A. Hage, R. N. Srinivasa, Diagn. Interv. Radiol. 2018, 24, 98.

[43] C. Kim-Fuchs, E. Angst, S. Vorburger, C. Helbling, D. Candinas, R. Schlumpf, Hernia 2012, 16, 21.

[44] M. J. Kieturakis, D. T. Nguyen, H. Vargas, T. J. Fogarty, S. R. Klein, Am. J. Surg. 1994, 168, 603.

[45] B. J. Ramshaw, J. G. Tucker, E. M. Mason, T. D. Duncan, J. P. Wilson, P. B. Angood, G. W. Lucas, Am. Surg. 1995, 61, 279.

[46] S. M. Maas, T. S. de Vries Reilingh, H. van Goor, D. de Jong, R. P. Bleichrodt, J. Am. Coll. Surg. 2002, 194, 388.

[47] S. L. Jackson, X. Tong, R. J. King, F. Loustalot, Y. Hong, M. D. Ritchey, Circ.: Heart Failure 2018, 11, e004873.

[48] N. Conrad, A. Judge, J. Tran, H. Mohseni, D. Hedgecott, A. P. Crespillo, M. Allison, H. Hemingway, J. G. Cleland, J. J. V. McMurray, K. Rahimi, Lancet 2018, 391, 572.

[49] J. R. Garcia, P. F. Campbell, G. Kumar, J. J. Langberg, L. Cesar, L. Wang, A. J. García, R. D. Levit, JACC: Basic Transl. Sci. 2017, 2, 601.

[50] E. B. Dolan, L. Kovarova, H. O’Neill, M. Pravda, R. Sulakova, I. Scigalkova, V. Velebny, D. Daro, N. Braun, G. M. Cooney, G. Bellavia, S. Straino, B. L. Cavanagh, A. Flanagan, H. M. Kelly, G. P. Duffy, B. P. Murphy, J. Biomater. Appl. 2018, 33, 681.

[51] S. M. Rossi, T. E. Murray, J. Cassidy, M. J. Lee, H. M. Kelly, CardioVasc. Intervent. Radiol. 2019, 42, 289.

[52] M. A. Horvath, C. E. Varela, E. B. Dolan, W. Whyte, D. S. Monahan, C. J. Payne, I. A. Wamala, N. V. Vasilyev, F. A. Pigula, D. J. Mooney, C. J. Walsh, G. P. Duffy, E. T. Roche, Ann. Biomed. Eng. 2018, 46, 1534.

[53] G. P. Duffy, T. Ahsan, T. O'Brien, F. Barry, R. M. Nerem, Tissue Eng., Part A 2009, 15, 2459

[54] E. T. Roche, M. A. Horvath, I. Wamala, S. E. Song, W. Whyte, Z. Machaidze, N. V. Vasilyev, D. J. Mooney, F. A. Pigula, C. J. Walsh, Sci. Transl. Med. 2017, 9, eaaf3925. 